\title{
Dextran Sulphate triggers platelet aggregation via direct activation of PEAR1
}

Christophe Vandenbriele ${ }^{a}$, Yi Sun ${ }^{b}$, Maarten $\mathrm{Criel}^{a}$, Katrien Cludts ${ }^{a}$, Soetkin van

Kerckhoven $^{a}$, Benedetta Izzi ${ }^{a}$, Thomas Vanassche ${ }^{a}$, Peter Verhamme ${ }^{a}$, Marc F. Hoylaerts ${ }^{a}$

${ }^{a}$ Center for Molecular and Vascular Biology, Department of Cardiovascular Sciences, Herestraat 49, bus 911, KU Leuven, Leuven, Belgium

${ }^{b}$ Cell Surface Signalling Laboratory, Wellcome Trust Sanger Institute, Cambridge CB10 1HH, United Kingdom 
Figure S1

AGGREGATION

A

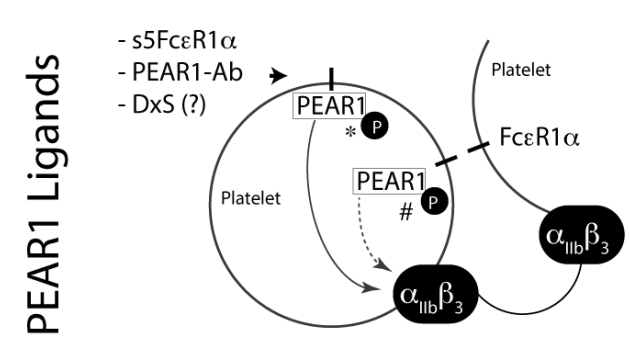

Direct $(*)$ and indirect (\#)

PEAR1-phosphorylation

B

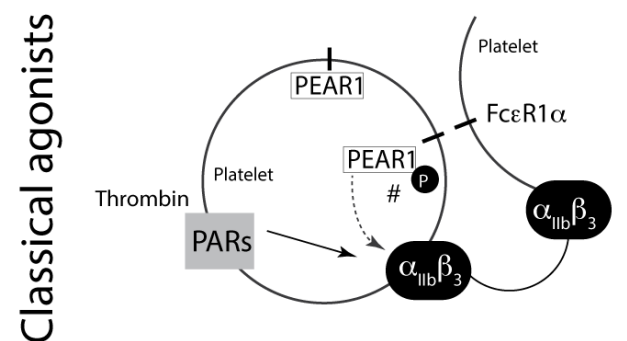

Indirect (\#)

PEAR1-phosphorylation

NO AGGREGATION

C Stirring + Eptifibatide

- s5FceR1 $\alpha$

- PEAR1-Ab

- DXS (?)

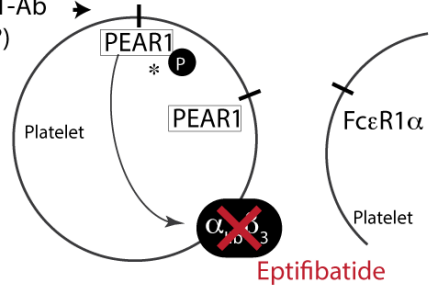

Direct $\left.{ }^{*}\right)$

PEAR1-phosphorylation

D

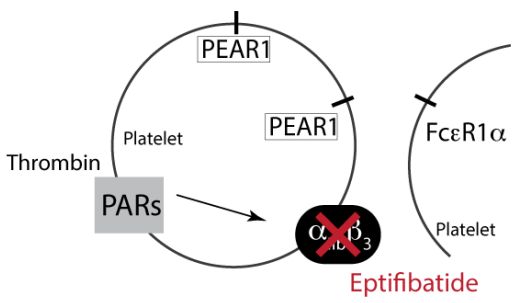

No

PEAR1-phosphorylation
E

Static

- s5FceR1 $\alpha$

- PEAR1-Ab $\rightarrow$

- DXS (?)

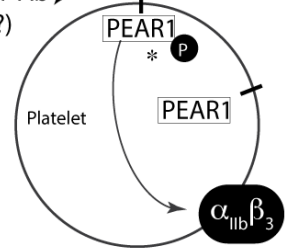

Direct $\left({ }^{*}\right)$

PEAR1-phosphorylation

F

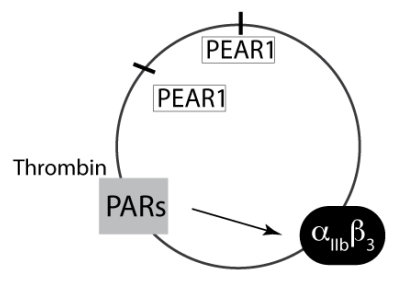

No

PEAR1-phosphorylation

G
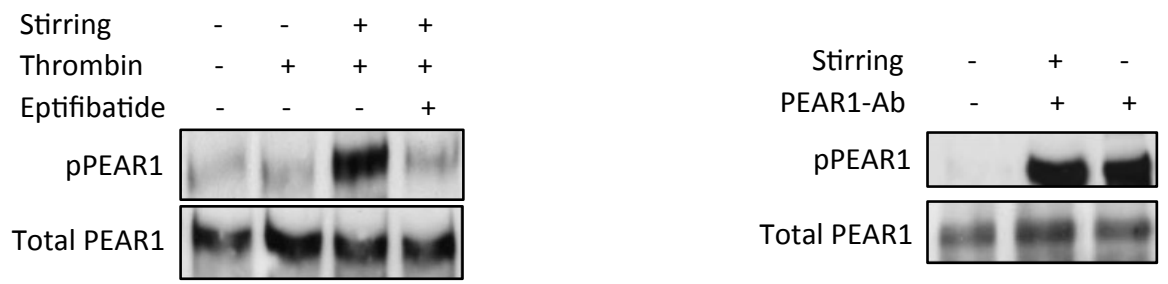


\section{Figure S1 - Platelet PEAR1-phosphorylation can be triggered directly or}

\section{indirectly}

This figure is crucial to illustrate direct activation of PEAR1 by DxS. As previously published by our group [3], PEAR1 phosphorylation can be the result of direct PEAR1-activation (* in Figure S1) via a PEAR1-ligand (e.g. soluble recombinant pentameric FceR1 $\alpha(\mathrm{s} 5 \mathrm{Fc} \varepsilon \mathrm{R} 1 \alpha)$ or anti-PEAR1-extracellular-antibodies) interaction or can be triggered indirectly (\# in Figure S1) as part of platelet amplification with traditional platelet agonists (e.g. thrombin, collagen). This has been schematically shown in Figure $\mathrm{S} 1$ and confirmed by western blot for pPEAR1 (PEAR1 immunoprecipitation and western blot for P-Tyr; Figure S1G). Direct activation of platelets by a specific PEAR1-ligand under stirring conditions (Figure S1A) results in direct PEAR1-phosphorylation via a PEAR1-ligand interaction and indirect PEAR1phosphorylation via platelet-platelet contact. This is in contrast with platelet activation under stirring conditions with classical platelets agonists (Figure S1B), where only indirect PEAR1-phosphorylation occurs via platelet-platelet contact. Incubation of platelets with a specific PEAR1-ligand in non-aggregating conditions (stirring conditions in the presence of eptifibatide (Figure S1C) or static conditions (Figure S1E)) results only in direct PEAR1-phosphorylation due to the lack of platelet-platelet contact. The presence of classical platelet agonists in non-aggregating conditions does not result in phosphorylation of PEAR1 due to the lack of direct PEAR1activation and the lack of platelet-platelet contacts. 